\title{
Hippocampal lesions and temporally chained conditioned stimuli in a conditioned suppression paradigm
}

\author{
ROBERT N. LEATON and GEORGE S. BORSZCZ \\ Dartmouth College, Hanover, New Hampshire
}

\begin{abstract}
Rats with lesions restricted primarily to the dorsal hippocampus were compared with shamoperated controls in a barpress conditioned suppression paradigm. Training on the barpress baseline involved temporally chaining different conditioned stimuli (CSs), each $30 \mathrm{sec}$ in duration, and began with $\mathrm{CS}_{1}$ followed by a shock unconditioned stimulus (US). When criteria for suppression were reached on $\mathrm{CS}_{1}-\mathrm{US}$, the animal was advanced to $\mathrm{CS}_{2}-\mathrm{CS}_{1}-\mathrm{US}$. Training progressed in this way to $\mathrm{CS}_{4}-\mathrm{CS}_{3}-\mathrm{CS}_{2}-\mathrm{CS}_{1}-\mathrm{US}$. The rats with hippocampal lesions learned $\mathrm{CS}_{1}-\mathrm{US}$ as rapidly as controls did, but they progressively failed relative to the controls as training progressed to the other CSs. This problem is interpreted as a temporal analogue of the spatial sequence in complex maze learning, and the lesion-induced deficit is interpreted as an inability to develop conditioned expectancies. We argue for an analysis of hippocampal deficits in terms of the established phenomena of conditioning.
\end{abstract}

One of the most prominent and consistent characteristics of rats with hippocampal lesions is a severe general deficit in complex maze performance. This deficit is exemplified by impaired performance on a wide variety of maze tasks, including the Maier three-table task (Rabe \& Haddad, 1969), plus mazes (Plunkett, Faulds, \& Albino, 1973), circular mazes (O’Keefe, Nadel, Keightley, \& Kill, 1975), radial arm mazes (Olton, Walker, \& Gage, 1978), and complex mazes of all descriptions and arrangements (see Gray, 1982; O'Keefe \& Nadel, 1978, for reviews). Although these maze tasks vary widely in their procedural details, it is impairment on these tasks that provides the cornerstone for O'Keefe and Nadel's (1978) spatial mapping theory of the hippocampus. The psychological roots of their theory lie with Tolman (1932), who proposed that rats solve these kinds of problems by creating cognitive maps of the environment. Historically, Tolman's cognitive interpretation of maze performance, which he considered to be based upon place learning, was contrasted with a stimulus-response (S-R), or responselearning, interpretation. The learning literature throughout the 1940s and 1950s was filled with attempts to find the experimentum crucis. Once it became clear that rats are neither exclusively place nor response learners (Restle, 1957), the issue for the S-R theorists was to account for the instances of learning that were obviously "place" learning. They did so by invoking some kind of anticipatory response mechanism, the prototype of which was

This research was supported in part by a grant from the Faculty Research Committee, Dartmouth College. We thank Tom Tighe and Fred Westbrook for their helpful criticisms of the manuscript. Requests for reprints should be addressed to R. N. Leaton, Department of Psychology, Dartmouth College, Hanover, NH 03755.
Hull's (1952) anticipatory goal response, $r_{\mathrm{g}}$. This conceptualization has taken many forms. It became the incentive factor for Spence (1956), anticipatory frustration, $r_{f}$, for Amsel (1958) to account for some aspects of extinction, and, finally, the anticipatory perceptual response, $r_{p}$ (Logan \& Wagner, 1965). Anticipatory response mechanisms clearly became the S-R theorists" "cognitive process," and the general theoretical mechanism to account for an animal's ability to act at a distance, to anticipate things outside its immediate environment.

Although details of the analysis have changed in contemporary learning theory, most of the analyses have in common with earlier treatments the assumption that Pavlovian conditioning processes generate anticipatory responses or expectancies that mediate instrumental behavior (Overmier \& Lawry, 1979). It is, therefore, according to this analysis, conditioned expectancies that guide a rat through a complex maze. (For the classical S-R analysis of maze learning, see Tighe, 1982.) The animal can anticipate events in the goal box (places where it has been rewarded or punished) through a chain of expectancies developed through Pavlovian conditioning. From this perspective, one could develop a nonspatial analogue of maze learning as a sequence of conditioned stimuli (CSs) culminating in positive or negative reinforcement. An animal first learns the relationship $S_{1}$-US (unconditioned stimulus; reward or punishment), then $S_{2}-S_{1}$-US, then $S_{3}-S_{2}-S_{1}-U S$, and so on. In a maze, $S_{1}$, $S_{2}, S_{3} \ldots S_{n}$ are progressively more removed from the goal spatially. The conditioning analogue allows us to remove the stimuli temporally rather than spatially from the goal.

In this interpretation, an animal with hippocampal lesions shows impaired performance in a complex maze, 
not because of any specifically spatial impairment, but because of an impairment in a conditioned mediating process. The more remote the goal, either spatially or temporally, the less performance is under the control of immediate goal-related factors, and the more essential these conditioned expectancies become as mediators of performance. The present experiment was designed to test this hypothesis by training rats to a chain of CSs in a conditioned suppression paradigm. On a barpressing baseline, animals were trained with $S_{1}$-shock (the US) until they met a criterion of suppression. They were then trained on $S_{2}-S_{1}-U S$, and then $S_{3}-S_{2}-S_{1}-U S$, and then $S_{4}-S_{3}-S_{2}-S_{1}$-US. This paradigm is a serial order arrangement similar to a higher order conditioning paradigm, except that the sequence always ended with the US.

In addition to testing the specific hypothesis outlined above, this experiment was also designed to demonstrate the value of a conditioning approach to an understanding of the behavioral effects of hippocampal damage. We hoped that by applying a conditioning analysis to a spatial learning task we could generate a new prediction and confirm it experimentally. We believe that the wellestablished and reliable phenomena of conditioning provide us with an analytical tool that concepts such as "cognitive maps" lack. This, of course, is exactly what the S-R theorists were saying to Tolman some 40 years ago (see Tighe, 1982).

\section{METHOD}

\section{Subjects and Surgery}

An initial experiment and an independent replication used a total of 41 experimentally naive male Sprague-Dawley rats that were approximately 120 days old at the time of surgery. Twenty-three randomly selected animals received bilateral lesions of the dorsal hippocampus and 18 served as sham-operated controls. They were individually housed and maintained on a 14:10 h light:dark cycle with ad-lib access to food and water. A minimum of 2 weeks after surgery, the rats were placed on a food-deprivation schedule that maintained them between $80 \%$ and $85 \%$ of their free-feeding weights. Each animal was fed its daily ration of food following its daily test session.

Surgery was performed under sodium pentobarbital anesthesia (45 mg/ $\mathrm{kg}$ ), and atropine sulphate $(20 \mathrm{mg} / \mathrm{kg}$ ) was used to reduce mucous secretions. Lesions were made electrolytically with electrodes stereotaxically oriented according to the atlas of Pellegrino and Cushman (1967). Two lesions were made on each side of the brain $2 \mathrm{~mm}$ posterior to bregma. One was $1 \mathrm{~mm}$ lateral to the midline and $3.3 \mathrm{~mm}$ below the dural surface, and the other was $2 \mathrm{~mm}$ lateral and $3.4 \mathrm{~mm}$ below the dural surface. At each site, an anodal current of $2 \mathrm{~mA}$ was used for a duration of $15 \mathrm{sec}$. Electrodes were constructed of 27-ga stainless steel hypodermic tubing insulated except for $0.5 \mathrm{~mm}$ at the tip. A head holder similar to that described by Frommer (1971) was used to avoid damage to the rats" eardrums. The sham-operated rats were anesthetized and placed in the stereotaxic instrument; their skulls were bared but not opened, and the wound was closed.

\section{Anatomical Procedures}

Following the experiment, the animals were intracardially perfused with normal saline followed by $10 \%$ formalin. The brains were soaked in a formalin-sucrose solution for at least $24 \mathrm{~h}$ before frozen sections were cut at $40 \mu \mathrm{m}$. Every fifth section was saved and stained by a modified Klüver-Barrera method. For evaluating the extent of the brain damage, the stained sections were projected onto brain diagrams taken from the atlas of Pellegrino and Cushman (1967). The damage was traced and reconstructed on diagrams separated by $0.2 \mathrm{~mm}$ throughout the full extent of the lesion and evaluated in terms of percentage of damage. To calculate this percentage, the area of damage to the hippocampus, including the dentate gyrus, was measured with a planimeter. The damaged area was compared with the total area of the hippocampus as represented on each of the brain diagrams. The total "volume" of the hippocampus was taken as the sum of the areas of the hippocampus shown on atlas diagrams from 0.8 to $5.6 \mathrm{~mm}$ posterior to bregma. The "dorsal hippocampus" was defined for descriptive purposes as the hippocampal area lying dorsal to the thalamus from 0.8 to $2.8 \mathrm{~mm}$ posterior to bregma. (The specification "dorsal hippocampus" is not meant to imply an anatomically or functionally meaningful distinction, and is used only to facilitate the description of the lesions.)

\section{Apparatus}

The animals were trained in one of four identical $29 \times 27 \times$ $25 \mathrm{~cm}$ operant chambers. The end walls were made of stainless steel and one had an operant bar and a recessed food cup. The top, front, and back were made of clear Plexiglas, and a sheet of milk glass covered the top. The floor was composed of $2-\mathrm{mm}$ stainless steel rods spaced $0.5 \mathrm{~cm}$ apart center to center. Each chamber was enclosed within a separate sound-attenuating chamber. A ventilation fan provided continuous background noise ( $72 \mathrm{~dB}$ SPL). Four different CSs were used. CS, was a 79-dB (SPL) white noise presented through a speaker in the end wall containing the bar and food cup. $\mathrm{CS}_{\mathbf{2}}$ was the onset in the otherwise dark chamber of a 7.5-W white light bulb mounted $12.5 \mathrm{~cm}$ above the chamber top. $\mathrm{CS}_{3}$ was an $89-\mathrm{dB}, 8-\mathrm{kHz}$ tone presented through a speaker in the back of the chamber. $\mathrm{CS}_{4}$ was the flashing of the 7.5-W light at a rate of $13 / \mathrm{sec}$. Each CS was $30 \mathrm{sec}$ in duration. The US was a $1-\mathrm{mA}, 0.5-\mathrm{sec}$ scrambled footshock produced by Grason-Stadler shock generators. All auditory intensities were measured with a General Radio Sound Level Meter, with the microphone centered inside the operant chamber.

\section{Procedure}

Barpress training. The animals were first trained in one session that combined magazine training, in which $45-\mathrm{mg}$ food pellets (Noyes) were delivered on a 30 -sec variable-time schedule (VT30), and continuous reinforcement (CRF) of the barpress response. This session continued until 50 barpresses had been made. Subsequent sessions advanced the animals from CRF to VI-15 to VI-30 to VI60 schedules of reinforcement for the barpress response. Each session lasted until the rat had received 100 pellets. By Day 5 of training, all animals had reached the VI-60 schedule, and they were maintained on that schedule in daily 100 -min sessions throughout the remainder of the experiment.

Unconditioned suppression. Performance had stabilized on the VI-60 schedule after 16 days of training, and unconditioned suppression to the four CSs was assessed in three sessions on consecutive days. During each session, each of the four CSs was presented four times in a balanced order on a VI 5.5-min schedule. All CS presentations were $30 \mathrm{sec}$ in duration. A suppression ratio was calculated by dividing the number of barpresses during each CS presentation by the sum of the barpresses during the 30 -sec period before CS presentation (Pre) and the number of barpresses during the CS $[\mathrm{CS} /($ Pre $+\mathrm{CS})]$. This ratio could range from 0 (complete suppression) to 0.5 (no suppression), with values above 0.5 indicating increased responding to the CS.

Conditioned suppression and chaining. Following the assessment of unconditioned suppression, conditioned suppression training began in daily 100-min sessions. In each session, four CS-US 
pairings were presented on a fixed 20 -min intertrial interval superimposed on the animal's ongoing baseline of barpressing. The first trial was presented $20 \mathrm{~min}$ into the session and the session ended approximately $20 \mathrm{~min}$ after the fourth trial. Training began with $\mathrm{CS}_{1}$ (white noise) followed by the US presented at CS offset. When criterion was reached on $\mathrm{CS}_{1}$, the light $\left(\mathrm{CS}_{2}\right)$ was presented prior to $C S_{1}$, which, in turn, was followed by the US. When criterion was reached on $\mathrm{CS}_{2}, \mathrm{CS}_{3}$ (the pure tone) was introduced in a similar manner, so that the sequence became $\mathrm{CS}_{3}-\mathrm{CS}_{2}-\mathrm{CS}_{1}-\mathrm{US}$. When criterion was reached on $\mathrm{CS}_{3}, \mathrm{CS}_{4}$ (the flashing light) was introduced, the final sequence becoming $\mathrm{CS}_{4}-\mathrm{CS}_{3}-\mathrm{CS}_{2}-\mathrm{CS}_{1}-$ US. All CSs were $30 \mathrm{sec}$ in duration, and in each case a subsequent CS came on at the offset of the prior CS. The US was always presented at the offset of CS,

An animal had to reach two criteria before the next-level CS was introduced. First, a suppression ratio of 0.15 or less on four out of five consecutive trials was considered an indication of significant suppression. If this was followed by a suppression ratio of 0.15 or less on six out of eight consecutive trials (a stability criterion), the next CS in the sequence was introduced. In addition, an animal had to maintain criterion suppression on all prior CSs and stable barpressing during the pre-CS period. A suppression ratio was calculated for each CS on each trial as described above for unconditioned suppression. The pre-CS period was the $30 \mathrm{sec}$ prior to the first CS in the series. A total of 37 days (148 trials) of conditioned suppression training were run.

Extinction. Following this chaining sequence, all animals were given two additional training sessions with four presentations of $\mathrm{CS}_{1}$-US in each session. These two "retraining" sessions were followed by 12 daily sessions ( 48 trials) of extinction in which $\mathrm{CS}_{1}$ was presented alone.

\section{RESULTS}

\section{Anatomical}

Diagrammatic cross sections of a representative hippocampal lesion are shown in Figure 1. The mean damage ( $\pm S E$ ) to the dorsal hippocampus (as defined above) was $74 \% \pm 3.36 \%$ and $17 \% \pm 1.05 \%$ to the total hippocampus.

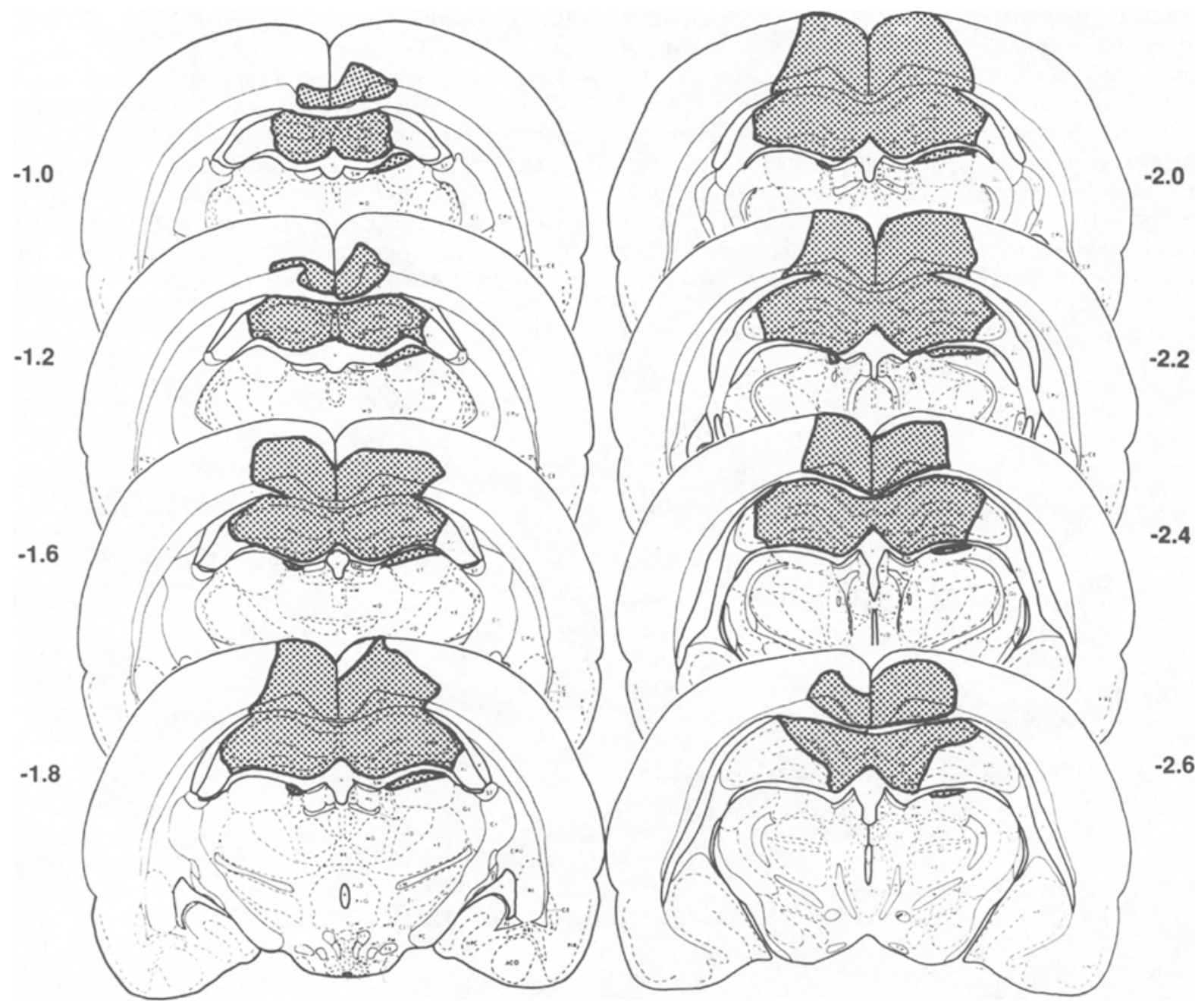

Figure 1. Diagrammatic cross sections of the lesion in a representative animal. The lesion shown destroyed $77 \%$ of the dorsal hippocampus and $16 \%$ of the total hippocampus (as defined in the text). The thalamic damage shown is from a different animal and is representative of the animals with thalamic damage. The sections are from the atlas of Pellegrino and Cushman (1967). The numbers are the coordinates (in mm) posterior to bregma. 
The damage closely approximated bilateral symmetry. In addition to the hippocampal damage, the cortex overlying the hippocampus, the subcortical white matter, and the corpus callosum were damaged in all animals. Twelve animals had some slight damage to the dorsal border of the thalamus, and in 9 of these animals the damage was bilateral throughout a portion of its course. The damage typically involved the anterior and lateral nuclei and less frequently the medial nuclei, the habenula, and the stria medullaris. Performance differences within the hippocampal-lesioned group were not correlated with variations in lesion size or placement.

\section{Barpressing}

The overall barpressing results from acquisition through the first 12 days of conditioned suppression training are shown in Figure 2. Both groups had reached an apparent asymptote of performance by the time unconditioned suppression was assessed (Training Days 17-19). The hippocampal group maintained higher daily response rates throughout all phases of the experiment. Analysis of Days $1-16$ yielded a significant group difference $[F(1,39)$ $=18.95, p<.001]$. Overall barpressing rates were suppressed to a comparable degree in both groups at the beginning of conditioned suppression training. Analysis over the 4 days before conditioned suppression training and the first 3 days of suppression training showed a significant change over days $[F(6,234)=8.08, p<.01]$ but no groups $\times$ days interaction $[F(6,234)=1.16$, $p>.30$ ]. Both groups had regained their prior response rates by about Day 8 of suppression training.

\section{Unconditioned Suppression}

The unconditioned suppression results are shown in Figure 3. The group differences are shown on the left side of the figure. Initially, the hippocampal group showed less unconditioned suppression than the controls. The group difference diminished over days as both groups habituated to the unconditioned effects of the stimuli. This pattern of results yielded a significant group difference $[F(1,39)=6.82, p<.05]$, a significant effect of days $[F(2,78)=88.91, p<.001]$, and a significant groups $X$ days interaction $[F(2,78)=9.85, p<.001]$. As shown on the right side of the figure, the stimuli differed in their potency to produce unconditioned suppression $[F(3,117)=9.95, p<.001]$, but the stimulus $\times$ groups interaction was not significant $[F(3,117)=1.37$, $p>.20$ ], indicating that the variation in stimulus potency was comparable for the two groups.

\section{Conditioned Suppression Training}

The performance in response to $\mathrm{CS}_{\mathbf{1}}$ (the white noise) is shown in Figure 4. Both groups acquired suppression rapidly and maintained stable performance thereafter with no apparent difference between the groups. Statistical analysis of the suppression ratios over these 12 trials showed no significant group difference $(F<1)$ or interaction $[F(11,429)=1.18, p>.20]$. Trials to criterion

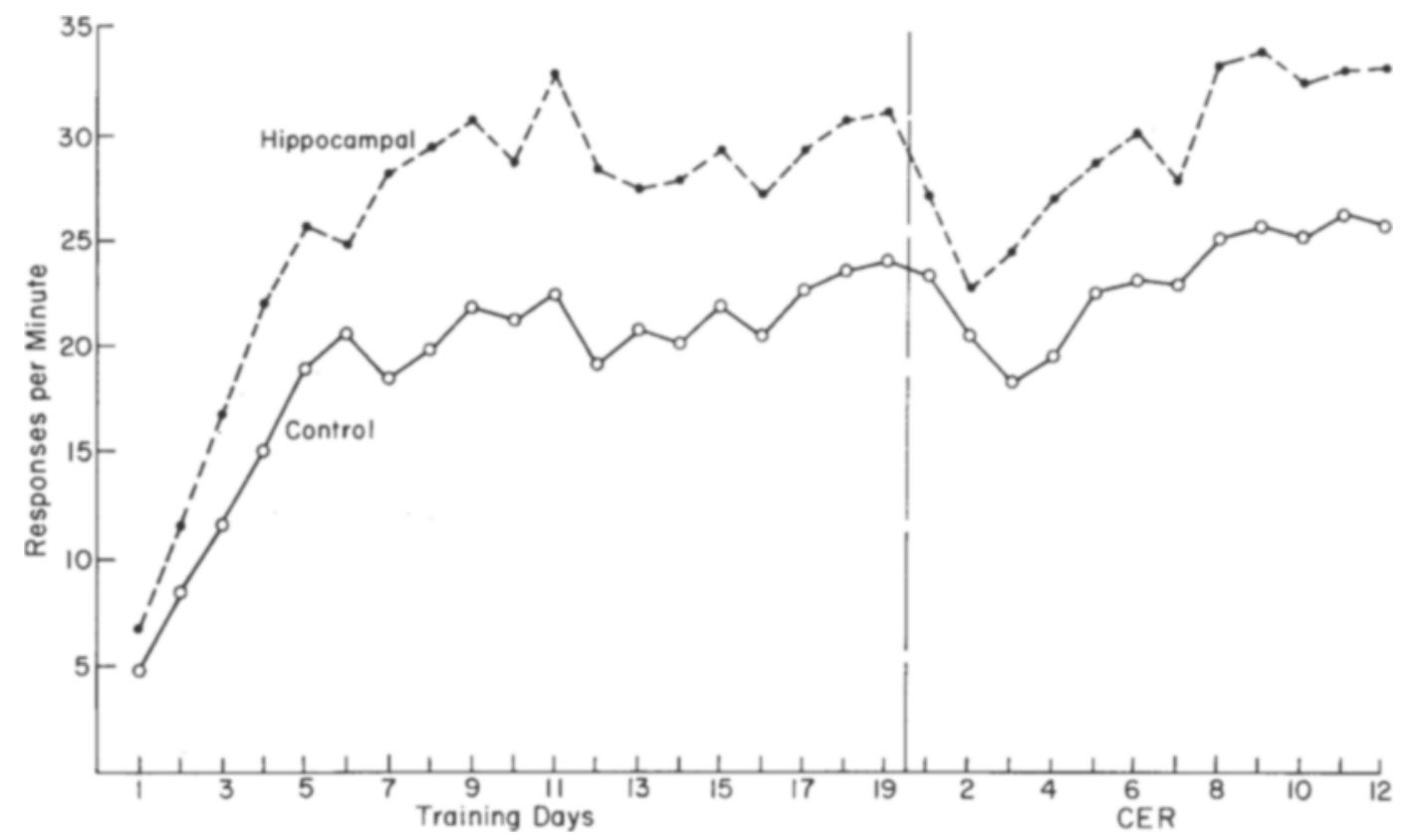

Figure 2. Barpress responses per minute for the hippocampal and control groups for the 19 days of acquisition (Training Days) and the first 12 days of conditioned suppression training (CER). All animals had reached the variable-interval 60-sec reinforcement schedule by Training Day 5. Unconditioned suppression was assessed on Training Days 17-19. 

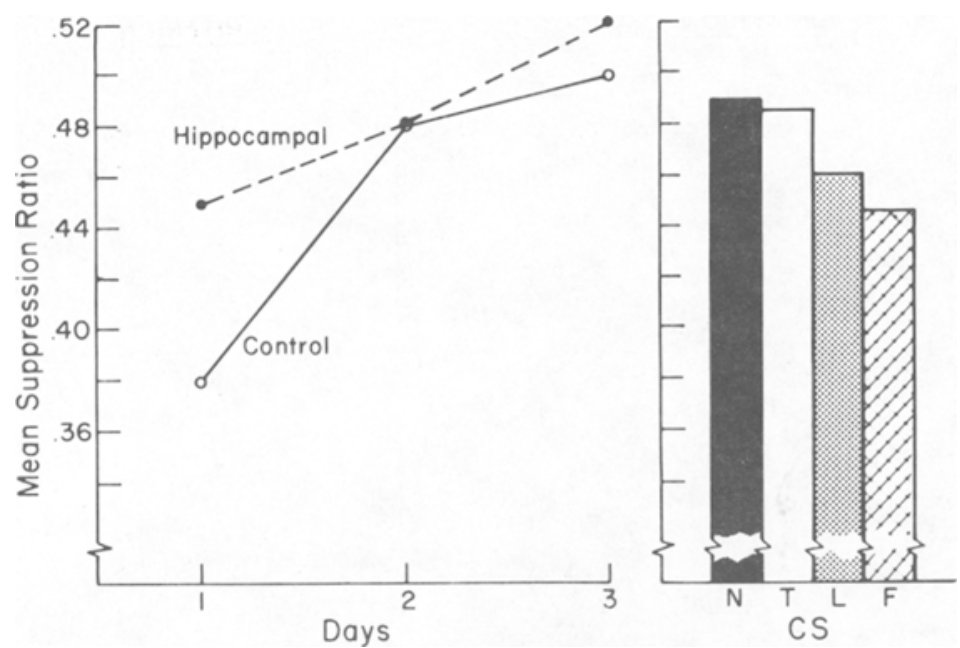

Figure 3. Mean suppression ratios during unconditioned suppression testing. The left side of the figure shows the mean dally suppression ratios for the hippocampal and control groups for the 3 test days collapsed across conditioned stimulus (CS) type. The right side of the figure shows mean suppression ratios for the four CSs collapsed across groups and days $(N=$ white noise, $T=$ tone, $L=$ light, $F=$ nashing light).

reflected the performance shown in the figure. Trials to Criterion 1 were 7.91 and 7.50 for the hippocampal and control groups, respectively, and to Criterion 2 they were 6.48 and 6.67, respectively (both $t \mathrm{~s}<1$ ).

Following $\mathrm{CS}_{1}$, trials to criterion and mean suppression ratios ceased to be meaningful measures for group comparison because a varying number of animals reached criterion on subsequent CSs and, therefore, a varying number of animals were performing on any given CS. The data were analyzed in terms of the percentage of animals that failed to reach the two criteria for the four CSs. These data are shown in Figure 5. As can be seen in the figure, the groups diverged as additional CSs were added to the sequence. Analysis of variance of these percentages for Criterion 1 over the four CSs yielded a significant group difference $[F(1,39)=19.64, p<.01]$ and a significant interaction of groups $\times \operatorname{CS}[F(3,117)=$ $4.80, p<.01]$. The same statistical pattern was found for Criterion 2 (both $p s<.01$ ). The addition of CSs in the sequence obviously made the task more difficult for

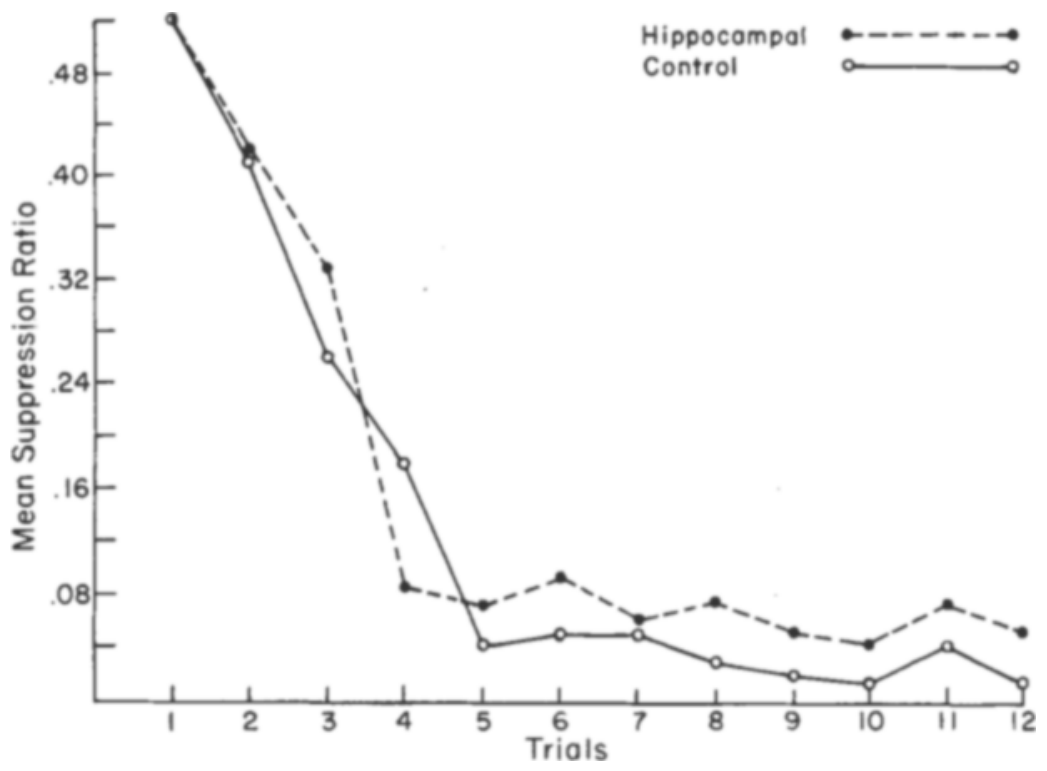

Figure 4. Mean suppression ratios for the hippocampal and control groups during acquisition in response to $\mathrm{CS}_{1}$ (white noise). 

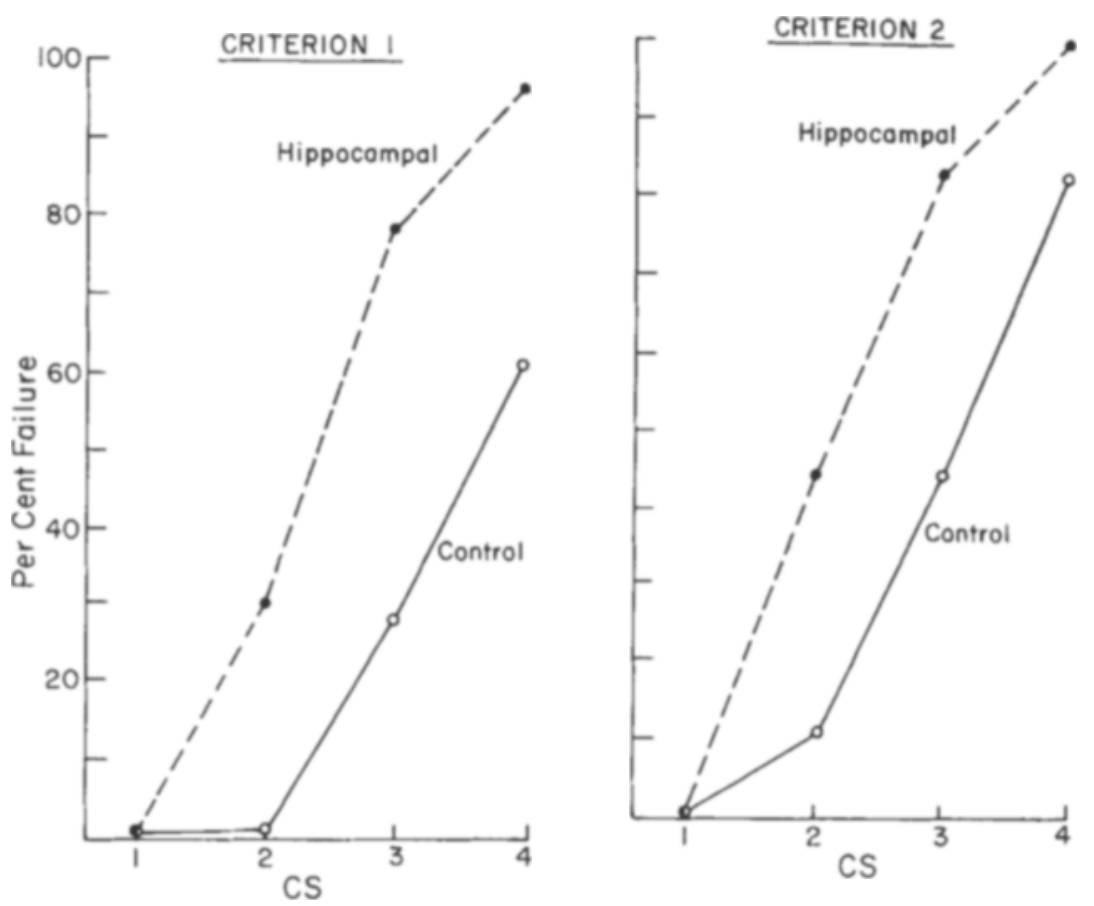

Figure 5. Percentage of animals in the hippocampal and control groups that failed to reach Criterion 1 (left) and Criterion 2 (right) as the chaining progressed from $\mathrm{CS}_{1}$ (white noise) to $\mathrm{CS}_{\mathbf{2}}$ (light) to $\mathrm{CS}_{3}$ (tone) to $\mathrm{CS}_{4}$ (flashing light).

both groups, but the effect was much more pronounced in the hippocampal group. Looking at just Criterion 1, the figure shows that all of the animals in both groups met the criterion on $\mathrm{CS}_{1}$, but although all of the controls met the criterion on $\mathrm{CS}_{2}, 30 \%$ of the hippocampal group failed to meet that criterion even with more than 130 training trials on the $\mathrm{CS}_{2}-\mathrm{CS}_{1}-\mathrm{US}$ problem. Only $39 \%$ of the controls reached criterion on $\mathrm{CS}_{4}(61 \%$ failed), but only 1 rat in the hippocampal group (4\%) reached criterion on this problem ( $96 \%$ failed).

In spite of the progressive failure on subsequent CSs in the series, all animals maintained performance on $\mathrm{CS}_{1}$ throughout training. Over the last 8 days of training, when some animals were performing on $\mathrm{CS}_{4}$ while some were still struggling to reach the first criterion on $\mathrm{CS}_{2}$, the mean suppression ratios on $\mathrm{CS}_{1}$ were .06 and .04 for the hippocampal and control groups, respectively $(t<1)$. It was striking to see animals in the hippocampal group which never approached criterion on $\mathrm{CS}_{2}$ after more than 130 trials still showing very low and stable suppression to $\mathrm{CS}_{1}$. Of the 7 hippocampal animals that failed to reach Criterion 1 on $\mathrm{CS}_{2}, 6$ were still performing at criterion levels on $\mathrm{CS}_{1}$ after 148 trials of training.

\section{Extinction of Suppression}

Figure 6 shows extinction of the suppression ratios to $\mathrm{CS}_{1}$. The hippocampal group extinguished significantly more rapidly than the controls. The group difference was significant $[F(1,38)=6.89, p<.05]$, as was the groups $\times$ days interaction $[F(11,418)=4.91, p<.001]$. (One animal in the control group died before the completion of extinction testing and is not included in the extinction data.)

\section{DISCUSSION}

Rats with lesions restricted primarily to the dorsal hippocampus, as compared with sham-operated controls,

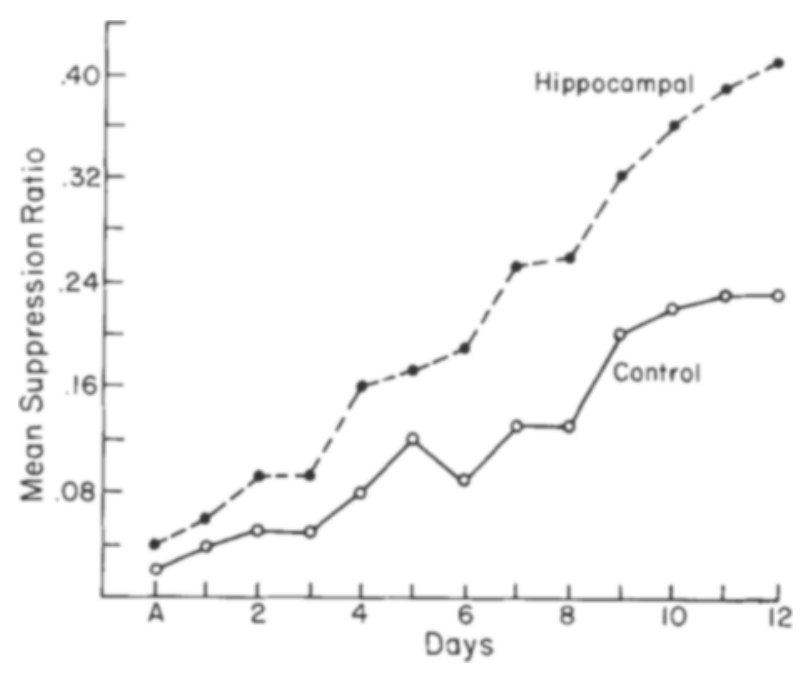

Figure 6. Mean daily suppression ratios for the hippocampal and control groups during the 12 daily extinction sessions to $\mathrm{CS}_{1}$. Each point during extinction is the mean of four daily trials. $A=$ mean suppression ratios for the two retraining sessions (eight trials) to $\mathrm{CS}_{1}$. 
progressively failed to maintain conditioned suppression performance on a temporally chained series of CSs. The hippocampal-lesioned animals acquired suppression to the first CS as readily as the controls did; they failed because they were unable to make the association from one CS to the next, and they progressively failed as the chained CS became temporally more remote from the shock US. We believe this temporal sequence is an analogue of the spatial sequence involved in complex maze learning, deficits in which are one of the hallmarks of rats with hippocampal lesions. The data support the hypothesis outlined in the introduction, that hippocampal lesions impair an animal's ability to develop conditioned expectancies, and that this impairment becomes more devastating for performance as the animal becomes more remote, whether spatially or temporally, from the goal.

Although pointing specifically to conditioned expectancies, the data do not address the basic nature of the underlying process. In theoretical terms, $\mathrm{CS}_{2}$ must be associated with $\mathrm{CS}_{1}$, or with a representation of the US, or with the conditioned response (see, e.g., Domjan \& Burkhard, 1986), but it is well beyond the scope of this paper to discuss the basic associative processes in Pavlovian conditioning. Whatever the precise nature of the association, it is clear that $\mathrm{CS}_{2}$, relative to $\mathrm{CS}_{1}$, is remote from the action of the US, and the representation of $\mathrm{CS}_{2}$ at the critical time of US presentation must be relatively weak compared with $\mathrm{CS}_{1}$. The further one moves back in the CS sequence, the weaker the specific CS representations become at the time of US application. Any manipulation that degrades the representation of the $C S$ in a conditioning paradigm might be expected to differentially impair the performance of animals with hippocampal lesions.

Hippocampal lesions typically do not impair first-order Pavlovian conditioning (see Gray, 1982; O'Keefe \& Nadel, 1978, for reviews), and acquisition to $\mathrm{CS}_{1}$ was not impaired in the present experiment. However, the lesioned animals extinguished conditioned suppression to $\mathrm{CS}_{1}$ more rapidly than did the controls. Extinction may be a more sensitive index of associative strength than acquisition, and this result may suggest weaker conditioning to $\mathrm{CS}_{\mathbf{1}}$ in the hippocampal-lesioned animals. Some weakness to the first CS could be expected since the mechanisms involved in anticipating $\mathrm{CS}_{1}$ or the US given $\mathrm{CS}_{2}$ may be the same, in process but not in strength, as those involved in anticipating the US given $\mathrm{CS}_{1}$. Any conclusion derived from the extinction data must be tempered, however, by the possible confounds associated with the difference in response rates between the groups and the differences in training experience the two groups received before beginning extinction.

This experiment was not designed as an attempt at yet another experimentum crusis, but rather as a test of a specific prediction derived from a specific theoretical analysis of performance in complex mazes. Thus, it is not surprising that the hypothesis may not readily apply to the complete corpus of hippocampal data, or that many hypotheses of hippocampal function could readily account for the data. Nothing would be gained by reviewing the entire empirical and theoretical literature at this time, but a few points should be made. Almost any form of an inhibition hypothesis of hippocampal function (e.g., Douglas, 1967; Kimble, 1968) would have no trouble with the data, and Gray's (1982) theory, which holds the hippocampus responsible for the generation of predictions, surely would find these data supportive. The present conceptualization has much in common with Rawlins's (1985) theory that considers temporal discontiguity as the critical variable in hippocampal lesion-induced impairments. Our emphasis, however, is not on time, per se, but rather on the mediating processes that allow an animal to bridge temporal intervals. Several recent experiments (e.g., Sutherland \& Rudy, 1988) have shown that the temporal interval itself is not the critical variable in at least some spatial learning deficits in hippocampal-lesioned rats. The present hypothesis may appear to be a restatement in animal learning-theory terms of the cognitive mediation hypothesis derived primarily from a consideration of human amnesic data (Warrington \& Weiskrantz, 1982; Weiskrantz, 1982). Indeed, we consider the Pavlovian conditioned process as the cognitive mediator.

The present data, however, are not consistent with all theories. It is not clear how one important contemporary account of hippocampal function-the spatial learning hypothesis (O'Keefe \& Nadel, 1978)-can handle these results, which derive from temporal rather than spatial variables. Of course, our experiment was designed with that particular theory in mind. It has been argued (Nadel \& Willner, 1980) that "place strategies" may become involved in Pavlovian conditioning through context conditioning. This argument points to the inverse relationship often found between conditioning to the context and conditioning to a discrete CS (e.g., Randich \& Ross, 1985), and suggests that animals with hippocampal lesions should show less context conditioning than do intact animals. In the present experiment, overall barpressing rates provided one index of context conditioning. There were no significant differences between the groups in the extent of suppression of the overall rates at the beginning of conditioned suppression training, nor in the recovery of these rates as training continued (see Figure 2). At least in some conditioning paradigms, this "context" argument also predicts stronger conditioning to the discrete CS in hippocampal-lesioned rats because of the reduced competition from context conditioning. Our results are not consistent with this prediction.

We believe that the importance of our results relates not so much to the specific outcomes but rather to the theoretical considerations that led to the experiment. By taking a conditioning conception of the effects of hippocampal lesions on complex maze tasks, we were able to predict, and then confirm experimentally, a new finding. A temporal analogue of maze performance yielded the familiar spatial learning effects of hippocampal lesions. We are persuaded that more progress will be made in un- 
raveling the mysteries of hippocampal function if the effects are analyzed in terms of the well-established and reliably measured phenomena of conditioning.

As in all experiments, there are certain aspects of the results that require comment. The stimuli were not counterbalanced, but it seems unlikely that the results are due to specific learning deficits associated with specific types and modalities of stimuli. The stimuli differed in unconditioned suppression potency, but the weakest stimulus on this dimension, the white noise, was $\mathrm{CS}_{1}$, on which the groups did not differ. Also, stimulus modality changed from $\mathrm{CS}_{1}$ to $\mathrm{CS}_{2}$ (the houselight), which was a more potent unconditioned suppressor than $\mathrm{CS}_{1}$. Stimulus modality changed again to $\mathrm{CS}_{3}$ and again to $\mathrm{CS}_{4}$ (the flashing light), which was the most potent unconditioned suppressor. Given these facts, it would require an elaborately complex hypothesis to explain the progressive learning failure in terms of unique learning properties of the various stimuli.

The animals with hippocampal lesions, as compared with controls, showed the anticipated higher barpress response rates on the VI-60 reinforcement schedule (Jarrard, 1965) and the equally unsurprising reduced initial unconditioned suppression (e.g., Kaplan, 1968). Either or both of these effects could have influenced the outcome in the direction found. One might have expected the influence of either factor to be revealed on acquisition to the first CS, but nothing in the present experiment allows us to rule out their impact on the results.

It must also be acknowledged that the hippocampal group was compared with a sham-operated control group rather than a lesioned control group. The hippocampal lesions routinely involved the overlying corpus callosum, cingulate gyrus, and neocortex, whereas other extrahippocampal damage was unilateral and/or inconsistent. Thus, the results could be a function of the extrahippocampal cortical damage or this damage in combination with the hippocampal damage. This cortical damage was relatively small, averaging less than $3 \mathrm{~mm}^{3}$. In our experience (Leaton, 1965, 1981), and in most of the published literature, cortical lesions of this size and even larger rarely produce any substantial difference when compared with sham-operated controls. However, the possibility does exist that the results are related to damage other than that to the hippocampus, and even the typical lesioned control group would not address the problem associated with combined damage.

\section{REFERENCES}

AMSEL, A. (1958). The role of frustrative non-reward in non-continuous reward situations. Psychological Bulletin, 55, $102-119$.

DOMJAN, M., BURKHARD, B. (1986). The principles of learning and behavior (2nd ed.). Monterey, CA: Brooks/Cole.

Douglas, R. J. (1967). The hippocampus and behavior. Psychological Bulletin, 67, 416-442.

Frommer, G. P. (1971). Modified nontraumatic headholder. Behavior Research Methods \& Instrumentation, 3, 225-226.
Gray, J. A. (1982). The neuropsychology of anxiety: An enquiry into the functions of the septo-hippocampal system. Oxford: Clarendon. Hull, C. L. (1952). A behavior system. New Haven, CT: Yale University Press.

JARRARD, L. E. (1965). Hippocampal ablation and operant behavior in the rat. Psychonomic Science, 2, 115-116.

KAPLAN, J. (1968). Approach and inhibitory reactions in rats after bilateral hippocampal damage. Journal of Comparative \& Physiological Psychology, 65, 274-281.

Kimble, D. P. (1968). Hippocampus and internal inhibition. Psychological Bulletin, 70, 285-295.

LEATON, R. N. (1965). Exploratory behavior in rats with hippocampal lesions. Journal of Comparative \& Physiological Psychology, 59, 325-330.

Leaton, R. N. (1981). Habituation of startle response, lick suppression and exploratory behavior in rats with hippocampal lesions. Joumal of Comparative \& Physiological Psychology, 95, 813-826.

LoGAN, F. A., \& WAGNER, A. R. (1965). Reward and punishment. Boston: Allyn \& Bacon.

Nadel, L., Willner, J. (1980). Context and conditioning: A place for space. Physiological Psychology, 8, 218-228.

O'KEEFE, J., \& NADEL, L. (1978). The hippocampus as a cognitive map. Oxford: Clarendon.

O'Keefe, J., Nadel, L., Keightley, S., \& Kill, D. (1975). Fomix lesions selectively abolish place learning in the rat. Experimental Neurology, 48, 152-166.

Olton, D. S., Walker, J. A., \& Gage, F. H. (1978). Hippocampal connections and spatial discrimination. Brain Research, 139, 295-308.

OVermier, J. B., \& LAWry, J. A. (1979). Pavlovian conditioning and the mediation of behavior. In G. H. Bower (Ed.), The psychology of learning and motivation (Vol. 13, pp. 1-55). New York: Academic Press.

Pellegrino, L. J., \& Cushman, A. J. (1967). A stereotaxic atlas of the rat brain. New York: Appleton-Century-Crofts.

Plunkett, R. P., Faulds, B. D., Albino, R. C. (1973). Place leaming in hippocampectomized rats. Bulletin of the Psychonomic Society, 2, 79-80.

RABE, A., \& HADDAD, R. K. (1969). Integrative deficit after hippocampal lesions? Proceedings of the American Psychological Association, 4, 213-214.

Randich, A., \& Ross, R. T. (1985). Contextual stimuli mediate the effects of pre- and postexposure to the unconditioned stimulus on conditioned suppression. In P. D. Balsam \& A. Tomie (Eds.), Context and learning (pp. 105-132). Hillsdale, NJ: Erlbaum.

Rawlins, J. N. P. (1985). Associations across time: The hippocampus as a temporary memory store. Behavioral \& Brain Sciences, 8 , 479-496.

Restle, F. (1957). Discrimination of cues in mazes: A resolution of the "place-vs-response" question. Psychological Review, 64, 217-228.

SPENCE, K. (1956). Behavior theory and conditioning. New Haven, CT: Yale University Press.

SUtherland, R. J., \& RUdy, J. W. (1988). Place learning in the Morris place navigation task is impaired by damage to the hippocampal formation even if the temporal demands are reduced. Psychobiology, 16, 157-163.

TIGHE, T. J. (1982). Modern learning theory: Foundations and fundamental issues. New York: Oxford University Press.

Tolman, E. C. (1932). Purposive behavior in animals and men. New York: Century.

Warrington, E. K. \& Weiskrantz, L. (1982). Amnesia: A disconnection syndrome? Neuropsychologia, 20, 233-248.

Weiskrantz, L. (1982). Comparative aspects of studies on amnesia. Philosophical Transactions of the Royal Society of London, Series B, 298, 97-109.

(Manuscript received August 18, 1989; revision accepted for publication December 20, 1989.) 